\title{
An Adaptive Blended Learning Approach in the Implementation of a Medical Neuroscience Laboratory Activities
}

\author{
Thomas I. Nathaniel $^{1}$ (1) - Asa C Black ${ }^{1}$
}

Accepted: 1 March 2021 / Published online: 8 April 2021

(c) This is a U.S. government work and not under copyright protection in the U.S.; foreign copyright protection may apply 2021

\begin{abstract}
Background The COVID-19 pandemic revealed existing gaps in the medical educational system that is heavily dependent on the presence of medical students and teachers in laboratory and class for instruction. This affects continuity in the implementation of the neuroanatomy component of the medical neuroscience laboratory activities during COVID-19. We hypothesized that pivoting wet laboratory neuroanatomy activities to online using an adaptive flexible blended method might represent an effective approach in the implementation of laboratory neuroanatomy activities during a pandemic.

Methods The current study describes an adaptive flexible blended learning approach that systematically mixes virtual face-to-face interaction activities with the online learning of brain structures, and the discussion of clinical cases. Learning materials are delivered through both synchronous and asynchronous modes, and Year 1 medical students learn neuroanatomy laboratory activities at different locations and different times. Student performances in the adaptive flexible blended learning approach were compared with the learning of similar activities during an in-person implementation of neuroscience laboratory activities.

Results The results of using this adaptive flexible blended learning approach provided an autonomous independent learning, self-study approach that broadened student performance such that we have more students scoring between 80 and $89 \%$, whereas the in-person learning resulted in most of the students scoring $>90 \%$ in the medical neuroscience laboratory activities.

Conclusion An adaptive flexible blended learning approach that combined virtual face-to-face instruction using digital technology with online learning of neuroscience laboratory activities provided a unique educational experience for Year 1 medical students to learn neuroscience laboratory activities during the COVID-19 pandemic.
\end{abstract}

Keywords Adaptive flexible blended learning $\cdot$ Neuroanatomy $\cdot$ Neuroscience $\cdot$ Wet laboratory activities $\cdot$ COVID-19

\section{Introduction}

In the wake of the shutdown of academic activities following the onset of the COVID-19 pandemic, we attempted to determine how to configure a neuroscience laboratory for medical students in a remote learning setting by converting wet neuroscience laboratory activities to an online format. The absence of wet neuroscience laboratory activities due to COVID-19 may have a lasting impact on student learning. This is because providing online cross-sectional or whole brain images may not provide medical students with an

Thomas I. Nathaniel

nathanit@greenvillemed.sc.edu

1 University of South Carolina School of Medicine-Greenville, Greenville, SC 29605, USA appreciation for the fabric of the anatomy of the brain [1]. The advances in technology may allow for online distance learning and may represent an important approach for the implementation of neuroscience laboratory activities during COVID-19 [2]. Therefore, despite the possible limitations of this approach, remote learning during a pandemic like COVID-19 provides an opportunity to ask important questions about the efficacy of purposely designed, mainly online neuroscience laboratory activities, and how these activities can be advanced to help medical students take control of their learning process as they progress in their medical career and in the future. If it is possible, medical students and teachers would undoubtedly prefer to keep on doing more whole-brain surface anatomy of identifying all the lobes, gyri, and dissecting the coronal and sagittal parts to identify subcortical structures in the wet 
neuroscience laboratory activities. However, being forced to think and provide a different platform for learning may actually be quite beneficial for both students and teachers. Therefore, online and virtual neuroscience laboratory activities during the COVID-19 crisis may serve many goals; for example, this approach might help investigate whether such online activities are effective in enhancing student performance compared with in-person wet laboratory neuroscience activities. Moreover, it is important to know whether these online and virtual neuroscience laboratory activities can be suitably integrated into the design of a medical neuroscience course that can be used to carefully guide students through a learning expedition, rather than simply being made available as another resource, for learning during a pandemic.

While the pandemic pushed many medical schools into different directions with innovative ideas in delivering neuroscience laboratory instruction in an online setting, it is very important to take advantage of the resources that are available to implement those new ideas. For instance, it is important to evaluate whether those new ideas or resources are effective. This is because while generating ideas is one great advantage of working with an integrated team of neuroscientists, transforming the good ideas to help medical students perform well in this challenging time may lead to important benefits for students. One of the adjustments for every neuroscience faculty member in this challenging time is learning to use digital technology and communicate to students entirely virtually in the implementation of neuroscience laboratory activities. Therefore, while there are challenges in implementing neuroscience laboratory activities during the shutdown, there are also opportunities to develop a unique model of blended learning that comprises the blending of online activities with digital technology. This model represented an adaptive blended learning that used digital technology infrastructure that supported online learning, where activities can be designed to adapt in response to an individual learner's needs [3-6].

Adaptive learning refers to a kind of responsiveness [7]; for example, the National Academy of Science interprets the phrase as "How people learn" or "learner-centered" and this responsiveness represents one of the fundamental qualities of effective learning [8]. Thus, adaptive learning builds on what is known about an individual learner to systematically adjust content or learning activities [3, 3-12]. Medical students are known to be quite sophisticated in using digital technology in an integrated medical curriculum [13-15]. In addition, they are also accustomed to selfstudy and working independently at their own pace [16-20]. Therefore, an adaptive blended learning approach will suit the particular needs of medical students, especially in the context of geographical location and during the COVID-19 pandemic. Consequently, knowledge of what is known about the technological skills of medical students in an integrated medical curriculum could subsequently be used to develop adaptive learning activities for medical students in a time of pandemic.

While it is important to view blended learning as a continuum with a form of distance learning, "fully online with no classroom teaching," at one end and "teaching with no technology at all on the other end [21,22]," there should be adaptability to reflect the goal of controlling the pace of learning, time, place, or geographic location especially during a pandemic [23]. In this context, an adaptive blended learning approach represented a fundamental shift in a neuroscience laboratory instruction method that blended digital technology with online activities to provide a unique learning experience in a time of pandemic. In the adaptive blended learning pedagogy, the digital technology replaced face-to-face learning with a virtual interaction, and the online activities gave students some element of control over their learning. This adaptation provided an individualized customized, fluid schedule allowing medical students to combine virtual learning $[24,25]$ with online activities for student-centered neuroscience laboratory instruction.

In this study, we describe the implementation of an adaptive blended learning pedagogy by pivoting brain dissection from in-person neuroscience laboratory activities to a modified adaptive blended learning approach that provides a virtual laboratory format of interactive neuroanatomy activities, clinical case discussion sessions, online images, and a virtual discussion platform that allowed interaction between faculty and students. We compared performance in summative examinations in the adaptive blended learning activities with previous academic performance when neuroscience laboratory activities were implemented through in-person neuroscience laboratory settings. This comparison allowed us to determine whether the adaptive blended learning of neuroscience laboratory activities enhanced student performance when compared with the results of in-person neuroscience laboratory activities.

\section{Methods}

\section{Participants}

A total of 102 first year medical students in years 2019 and 2020 at the University of South Carolina School of MedicineGreenville who took the medical neuroscience course also participated in the neuroscience laboratory activity. In 2019, there were 57 female students, compared with 46 male students. In 2020, there were 58 female students, compared with 46 male students involved in the adaptive blended learning neuroscience laboratory activities. Average class age for both years was 23.0. The average score for admitted students on the Medical College Admission Test (MCAT) score for 
2019 was at the 71 st percentile, while that of 2020 was at the 73rd percentile. The average GPA for admitted students in 2019 was 3.66, while the average GPA for 2020 was 3.65. The study was approved by the Institutional Review Board (IRB), and all participants consented to participate.

\section{Description of the Typical Wet Neuroscience Laboratory Activities and Clinical Case Discussion}

Medical neuroscience is a 4-week course taught to medical students during the second semester of the first year after the Structure and Function 1 and 2 modules. In the implementation of the course, there were a variety of learning activities including active lectures, class discussions, small group sessions, clinical case presentations, and laboratory sessions in the form of cadaveric dissection. For the laboratory activities, medical students spent a total of $6 \mathrm{~h}$ during wet neuroanatomy laboratory activities in mandatory sessions. Students were able to spend additional time outside of the mandatory sessions for individual laboratory review of materials. The neuroanatomy laboratory portion of the course included detailed examination of the surface anatomy of the brain, dissection to identify subcortical structures, clinical case discussion, and review of neuroimaging in both coronal and sagittal sections. For the implementation of each laboratory session, there was a general description and an overview of specific objectives for the session, and students were provided with instructions for each of the laboratory activities (Table 1). The course assessment was comprised of five laboratory quizzes that accounted for $12 \%$ of the final grade, a laboratory and clinical correlation examination that accounted for $36 \%$ of the final grade, and a written examination that accounted for $52 \%$ of the final grade. In general, the laboratory component of the course comprised $48 \%$ of the total grade. During the dissections, a group of six to eight students shared a cadaver and rotated turns to dissect the brain under the supervision of neuroscience faculty (Neuroscience) and clinicians (Neurologists and Neurosurgeons). An important element of the neuroscience laboratory activity was the clinical case presentation sessions. Clinical cases were built on structures identified in the wet neuroanatomy laboratory to further reinforce the clinical relevance of specific neuroanatomical targets during the neuroscience clinical case discussion session. During these presentations, students were divided into small groups, and each group was provided with a clinical case that related to a component of neuroanatomy that was learned during a neuroanatomy wet laboratory experience. Students were provided with an opportunity to discuss the cases among their small groups and solve the problems by identifying the effect of specific lesions on specific structures based on the patient's clinical presentation. After the small group meeting, students then presented their analysis of the case to the whole class and answered related clinical questions pertaining to the clinical problem. For the graded examinations, students were tested on their knowledge of how to identify gross anatomical structures and radiological images in addition to answering clinical vignette-style questions in the effect of lesions on the patient's signs, symptoms and on the result of clinical tests.

\section{Description of Adaptive Blended Learning Activities}

At the University of South Carolina School of Medicine Greenville, the neuroscience laboratory activities were changed to online instruction in the 2020 academic year due to the COVID-19 pandemic that resulted in the shutting down of universities. While there were challenges in the adjustment, we implemented the same laboratory activities and volume of materials that we have maintained over the years. The only change for this year is that all activities were accomplished online through the adaptive blended learning model.

For the laboratory portion of the course which required detailed examination and identification of structures in the surface anatomy of the brain and the anatomy of subcortical structures, students were provided with neuroanatomical interactive virtual activities. Implementation of each laboratory session in the adaptive blended learning model involved a general description and an overview of specific objectives for the session, and students were provided with instructions for each of the laboratory activities.

From the student perspective, the neuroanatomical interactive virtual activities shifted all the lab activities, contents, and instructions to the control of the student allowing each student to manage the pace with the ability to pause, go back, or skip forward through online content as he or she wanted during implementation of the different activities. The interactive online activities replaced the traditional handson-laboratory neuroanatomical activities that had been the mainstay of our neuroscience laboratory activities over the years for year one medical students. The neuroanatomical interactive virtual activities were implemented by students using the "Digital Neuroanatomy" software developed by Leichnetz [53]. During the online activities, students were able to interact with several human brain dissections with the virtual brain, which can be resized and rotated in all directions, and certain areas can be selected allowing students to identify structures of particular interest. Moreover, students could select the particular dissection of interest and view the brief animated gifs of the actual dissections which demonstrated how each virtual brain was prepared. Multiple learning formats were provided comprising written instructions, video tutorials, and demonstrations of brain dissection, offering students a self-paced, interactive review of structures and functions of the brain. In addition, practice questions were provided to help apply learned concepts 
to clinical application. In most cases, students chose the time at which they performed their neuroscience laboratory activities in their different locations. This enabled faculty members to have time to interact with each student through email communication and the online interactive discussion platform on Canvas.

During virtual interactions, faculty members appeared in-person in an empty classroom (Fig. 1) to discuss clinical cases using WebEx/Panopto, allowing virtual interactions with students and the opportunity to respond to student questions. During these presentations, each clinical case was related to a component of neuroanatomy and allowed students to solve the problems by identifying the effect of specific lesions on specific structures based on the patient's clinical presentation. Therefore, clinical cases were built on structures that students identified in their online interactive activities concerning the identification of surface and subcortical structures. While the clinical sessions were led by faculty members, students participated virtually in the clinical case sessions through WebEx and asked questions for clarification of specific clinical concepts.

After the presentation, a detailed description of each clinical case presentation was posted online on Canvas for students, and all cases were recorded on Panapto/WebEx and posted online an hour after each case presentation. Transferring the recorded case discussions online did much of the heavy lifting in terms of saving time which would have been used in creating small group activities and delivering clinical case sessions in the classroom. The time saved permitted providing support on an as-needed basis through individual interactive tutoring sessions on the Canvas platform. This provided additional opportunity for students to review essential content, ask questions, and go over problems at their own pace. Therefore, the adaptive blended learning model offered our faculty members and students increased flexibility because online learning was helpful in delivering laboratory neuroanatomy instruction while digital technology played a major role in the clinical case discussion. The Canvas platform allowed faculty members to track student activities and progress in understanding of materials in the clinical case discussion and online activities. In general, we blended the online interactive neuroanatomy activities with virtual or live interactions to provide an integrated learning experience with appropriate level and topic for all activities in the neuroscience laboratory.

\section{Data Analysis}

Students had a weekly laboratory quiz assessment during all 4 weeks of the neuroscience module. The format of the laboratory quizzes involved identification of surface anatomy and subcortical structures using the neuroanatomy interactive software and clinical vignette style questions similar to the format used by questions on the National Board of

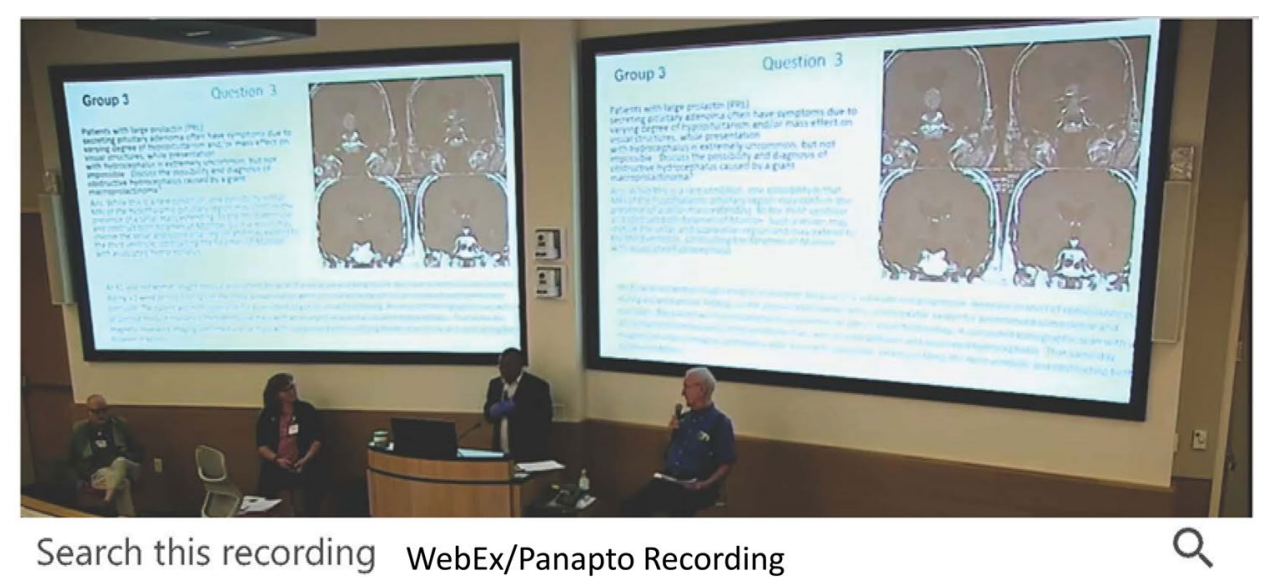

Discussion

Notes

Bookmarks

Fig. 1 The empty class room is shown while faculty members meet to discuss clinical cases and interact with students virtually. Panapto and WebEx platforms were very effective for live or virtual interaction in the discussion of clinical cases. This was very helpful in providing clinical relevance to learned neuroscience concepts and in the periodically interactive discussion platform. The different cases were discussed by faculty members to further reinforce the clinical relevance of specific neuroanatomical targets identified by students in their online activities. During the clinical case discussion by faculty members, online discussions on the Panapto and WebEx platform were also ongoing allowing virtual interaction with students, and real-time. Each session was recorded on Panopto and sent to students one hour at most after each lecture 


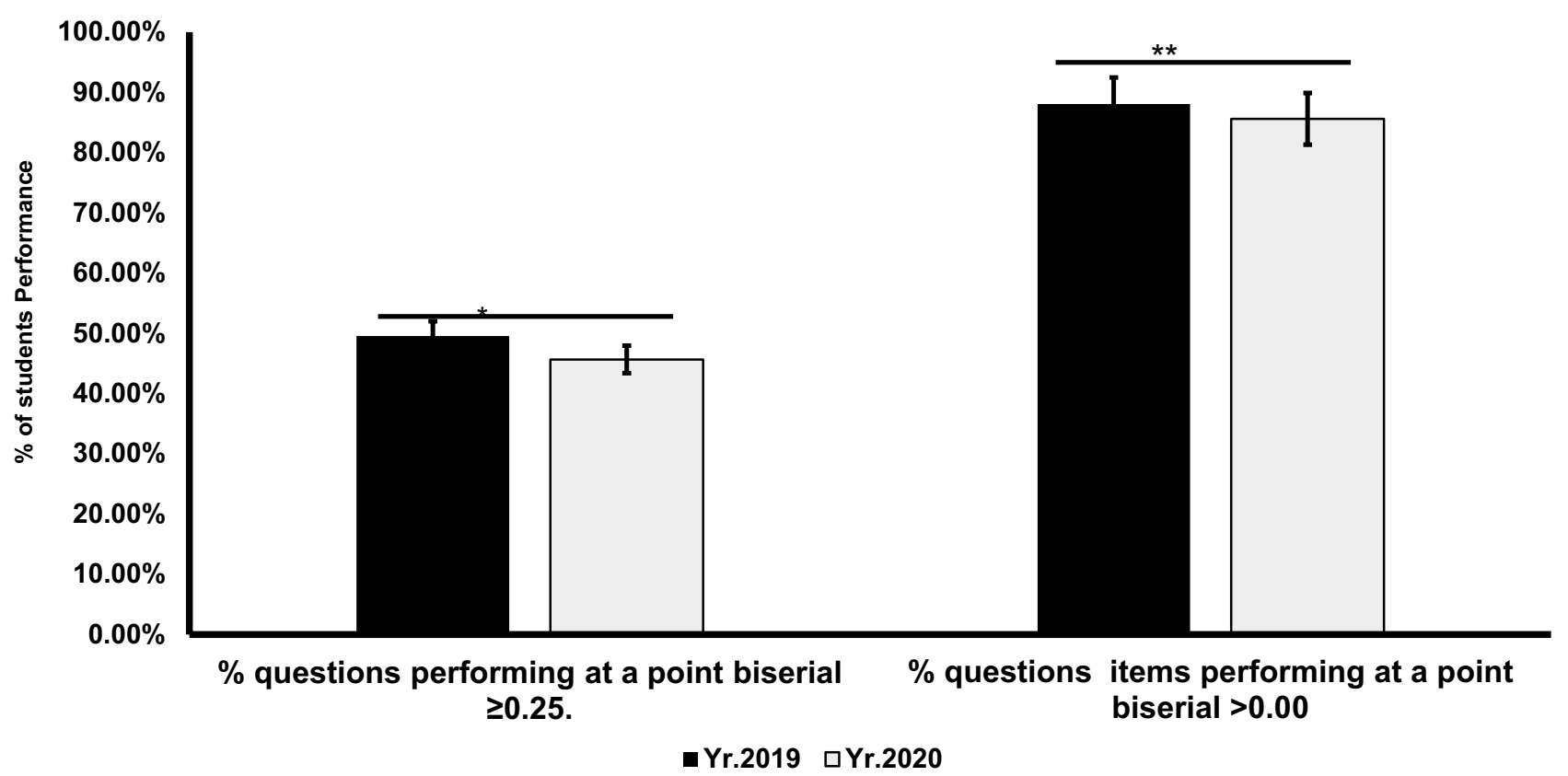

Fig. 2 Item performance measures for summative questions during the in-person (2019) and the adaptive blended learning sessions (2020)

Medical Examiners (NBME) STEP 1 Examination. The clinical vignette questions tested materials in the clinical case discussion sessions that were recorded on Panopto/ WebEx and posted on Canvas for students. We used the same examination format for the laboratory quizzes and the final laboratory exam. All proctoring was done using proctoring software developed by Examsoft Worldwide INC was used. The same set of questions used in 2019 were also used in 2020 for all examinations. The format for standard item analysis including the difficulty index and biserial values were calculated for each of the test questions. This analytical tool evaluated the performance of questions used on both laboratory quizzes and the final laboratory examination. The reliability of the examination was determined using the KR20 (Kuder-Richardson Formula 20) which is an index of the internal consistency reliability [26]. Values for KR20 generally range from 0.0 to 1.0 , with higher values representing a more internally consistent and reliable test capability of discriminating between students who had a higher understanding of the test material versus those with a poorer understanding. A KR20 value of 0.5 or higher is a good threshold value for determining whether or not a test is reliable [27]. The KR20 is calculated using the equation below;

$\mathrm{KR} 20=\frac{k}{k-1} \mathrm{x}\left(1-\frac{\sum_{i=1}^{k} \text { piqi }}{\delta^{2} x}\right)$ and $\delta^{2} x=\frac{1}{n} \sum_{i=1}^{n}(X i-\mu)^{2}$

All summative questions were mapped to each of the module level objectives. The mean percentage of performance for each of the module level objectives was computed for 2019 during wet lab neuroanatomy laboratory activities and in-person small group clinical case discussion and for 2020 involving flexible blended learning of all the materials. Normality of data distribution was determined using ShapiroWilk's test $(P<0.05)$. Student's $t$-test (two-tailed) was

Table 1 Learning objectives for the integrated medical neuroscience course

\begin{tabular}{|c|c|}
\hline Obj \# & Learning objectives \\
\hline 1 & Explain the biochemical processes that are important in the integrated functioning of the nervous system in complex \\
\hline $\begin{array}{l}2 \\
3\end{array}$ & $\begin{array}{l}\text { Describe the development and structure of major components of the nervous system at the macroscopic, microscopic, and } \\
\text { molecular levels } \\
\text { Correlate the structure of essential components of the nervous system to their physiological functions }\end{array}$ \\
\hline 4 & Identify and correlate clinical gross anatomical features of the nervous system using common analytic and imaging modalities \\
\hline 5 & Identify cross sectional anatomical features of the nervous system \\
\hline 6 & Correlate spatial relationships and orientation of anatomical features of the nervous system \\
\hline 7 & Explain clinical observations following lesions of structures and functional pathways of the nervous system \\
\hline
\end{tabular}


used to determine the significance of differences between 2019 and 2020 for the final summative laboratory examination. This analysis compared differences in student performance between the adaptive only blended learning and in-class clinical case discussion or wet neuroscience laboratory activities. Since student performances were spread between $70-79 \%, 80-89 \%$, and $>90 \%$ for both classes, we used ANOVA with repeated measures or within-subject and this enabled us to make broad comparisons for $70-79 \%$, $80-89 \%$, and $>90 \%$ for both classes. Post hoc analysis was used to determine significant differences between the performance categories in the adaptive blended learning and the in-person activities. Statistical significance was established at $P<0.05$. All statistical analyses were performed utilizing SPSS statistical package, version 25.0 (SPSS Inc., Chicago, IL).

\section{Results}

The results for the biserial analysis of students' performance in the test questions are presented in Fig. 2. The results indicate high discrimination for test questions for the final summative exams for both years, and they were not significantly different $(*, * * P<0.05)$. The results for the reliability (KR20) of the laboratory quizzes and final examinations are shown in Table 2. As shown in the table, KR20 values for the laboratory quizzes ranged between 0.18 and 0.69 for 2019 and 0.26 and 0.52 for 2020 . The KR20 value was higher in 2020 (0.80) than 2019 (0.70), indicating the stronger reliability of the final laboratory examinations in the adaptive blended learning sessions when all activities were delivered online. The mean score achieved in the examinations was broken down by each of the seven objectives and presented in Fig. 3. A direct comparison using the two-tailed, paired Student's $t$-test revealed that students' performances on objectives $1,2,3,4$, and 5 were significantly higher in $2019(P<0.001)$ when compared with 2020 . However, objectives 6 and 7 were not significantly different $(P>0.05)$ for 2019 and 2020. A direct comparison of student performance between 2019 and 2020 revealed a higher

Table 2 The Kuder-Richardson Formula 20 (KR20) tests for the reliability of the formative examinations. The formative examinations had KR20 values that range between 0.19 and 0.90 for 2019 and 0.58 performance in $2019(M=92, S D=0.15)$ when compared with $2020(M=90, S D=0.11), t(101)=2.66, P=0.009)$. ANOVA with repeated measures found a significant difference among students in the different performance categories between $70-79,80-89$, and $>90(F(2,399)=9.996$, $P=0.0471)$. Post hoc analysis revealed a significant difference $(P<0.05)$ among students in performance categories for 2019 and 2020 (Fig. 4).

\section{Discussion}

Three major findings arise from this study. First, the KR20 value was higher in 2020 than 2019, indicating the strength and reliability of the final laboratory examinations in the adaptive blended learning method when all activities were delivered online compared with the in-person learning of neuroscience laboratory activities. Also, the biserial analytical tool revealed high discrimination, and an acceptable level of difficulty. This tool provided a suitable mechanism for validating questions and testing the performance of the same questions used in 2019 and 2020 examinations. Second, student performance was significantly higher in six of the seven module objectives for 2019 when compared with 2020. Third, students' performances in the final laboratory summative examination were higher in 2019 than 2020. This result suggested a better performance in the in-person neuroscience laboratory activities. Finally, the stronger performance in 2019 was mainly due to $70 \%$ of the students scoring $>90 \%$, whereas in 2020 , the scores were more broadly distributed between students that scored $80-89 \%$ and $>90 \%$. These findings reveal that the in-person neuroscience laboratory activities which involved the dissection of the brain during wet neuroanatomy laboratory activities and small group discussion of clinical cases were associated with a better performance when compared with the adaptive blended learning of all the materials.

In 2019 (in-person learning), 70\% of the students scored $>90 \%$ while $2 \%$ scored between 70 and 79 . Students who scored $>90 \%$ in the summative examination reflect high performing students, and the in-person wet laboratory

and 0.88 for 2020 . The scores for both years were high, indicating the strong reliability of the formative examinations

\begin{tabular}{llllll}
\hline & $\begin{array}{l}\text { Yr 2020 } \\
\text { Mean } \pm \text { S.E }\end{array}$ & $\begin{array}{l}\text { Yr 2019 } \\
\text { Mean } \pm \text { S.E }\end{array}$ & $P$-value & 2019 KR 20 & 2020 KR 20 \\
\hline Quiz 1 & $98.12 \pm 0.13$ & $99.36 \pm 0.16$ & $>0.05$ & 0.19 & 0.28 \\
Quiz 2 & $99.13 \pm 0.17$ & $99.37 \pm 0.24$ & $>0.05$ & 0.18 & 0.26 \\
Quiz 3 & $94.76 \pm 0.15$ & $94.29 \pm 0.16$ & $>0.05$ & 0.61 & 0.52 \\
Quiz 4 & $96.98 \pm 0.21$ & $77.30 \pm 0.24$ & $<0.05$ & 0.63 & 0.29 \\
Quiz 5 & $93.97 \pm 0.20$ & $91.63 \pm 0.18$ & $<0.05$ & 0.34 & 0.33 \\
\hline
\end{tabular}




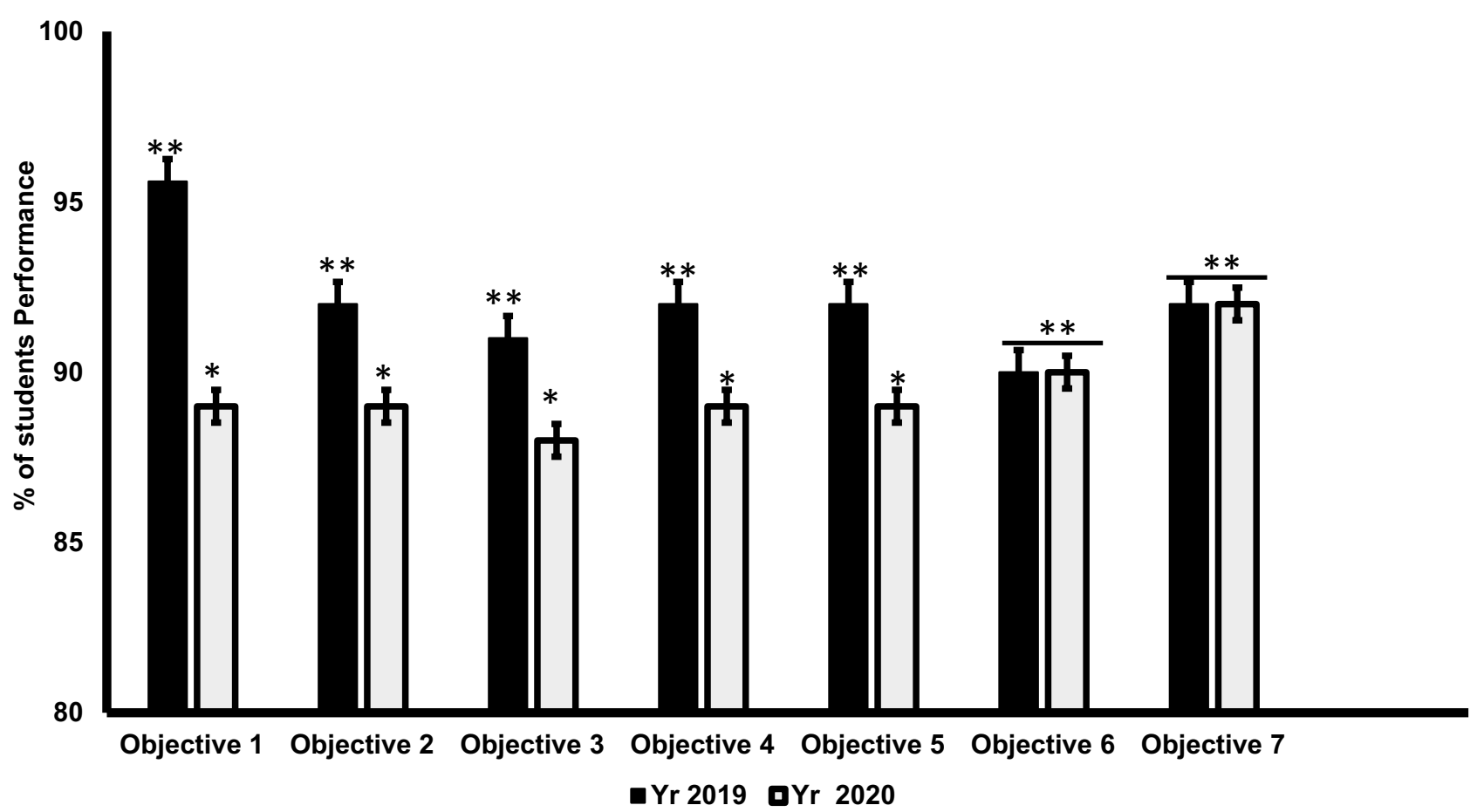

Fig. 3 Percentage mean scores achieved on the summative examinations for each of the eight objectives. Number of medical students in the 2019 ( $n=102)$ during the blended learning activities compared

neuroscience activities and clinical case discussion session format may have been more favorable to these sets of students. In the in-person neuroscience laboratory activities, students applied the information they learned in the neuroscience lecture sessions about the anatomy of the brain to an actual cadaveric brain. They worked together in small groups, interacted, and discussed with one another about identified different structures on the cadaveric brain. In addition, during the weekly wet neuroanatomy laboratory session that involved both biomedical science faculty members and clinicians, students had the opportunity to interact with clinicians who shared clinical cases related to the neuroanatomy of structures observed on the cadaveric brains. Clinicians analyzed clinical cases that they diagnosed related to specific structures learned and highlighted their clinical relevance. Therefore, analyzing clinical cases also during cadaveric brain dissection in the wet neuroanatomy laboratory activities probably provided a suitable format for high-performing students especially in processing-related clinical neuroscience concepts. Medical students are specifically known to show preference to learning materials that emphasize clinical relevance that they deem relevant to their future careers [28-30]. Therefore, it is possible that the presence of clinicians in the dissection laboratory during the wet neuroanatomy laboratory activities was very helpful in emphasizing the clinical relevance of identified structures. Moreover, the with 2020 adaptive flexible blended learning of all materials in an online format $(n=102)$

reinforcement of concepts that occurred through the identification of structures in the wet neuroanatomy laboratory activity during the weekly clinical correlates or case discussion sessions presented by biomedical science faculty members played a major role in further simplifying the learned concepts. This reinforcement enables the immediate learning of the association between structures and its applicability to a particular neurologic disease [31-37]. This format was probably very helpful to low- and high-performing students resulting in more students scoring $>90 \%$ and fewer students scoring less than $70 \%$ in the final neuroscience laboratory examination.

While the in-person neuroscience laboratory activities that included wet neuroanatomy laboratory and clinical case discussion sessions produced more students with very high scores, the adaptive blended learning produced more categories of students with $70-79 \%$ and those that scored $80-89 \%$. The major feature of the adaptive blended learning is that it allowed students to move on an individually customized, fluid schedule for learning activities. The approach also provided a virtual interactive learning environment to replace the face-to-face interaction with students. Therefore, while medical students were able to operate independently and control the pace of their learning neuroscience laboratory activities during a pandemic, they also received help from biomedical science faculty through WebEx and Panopto to 


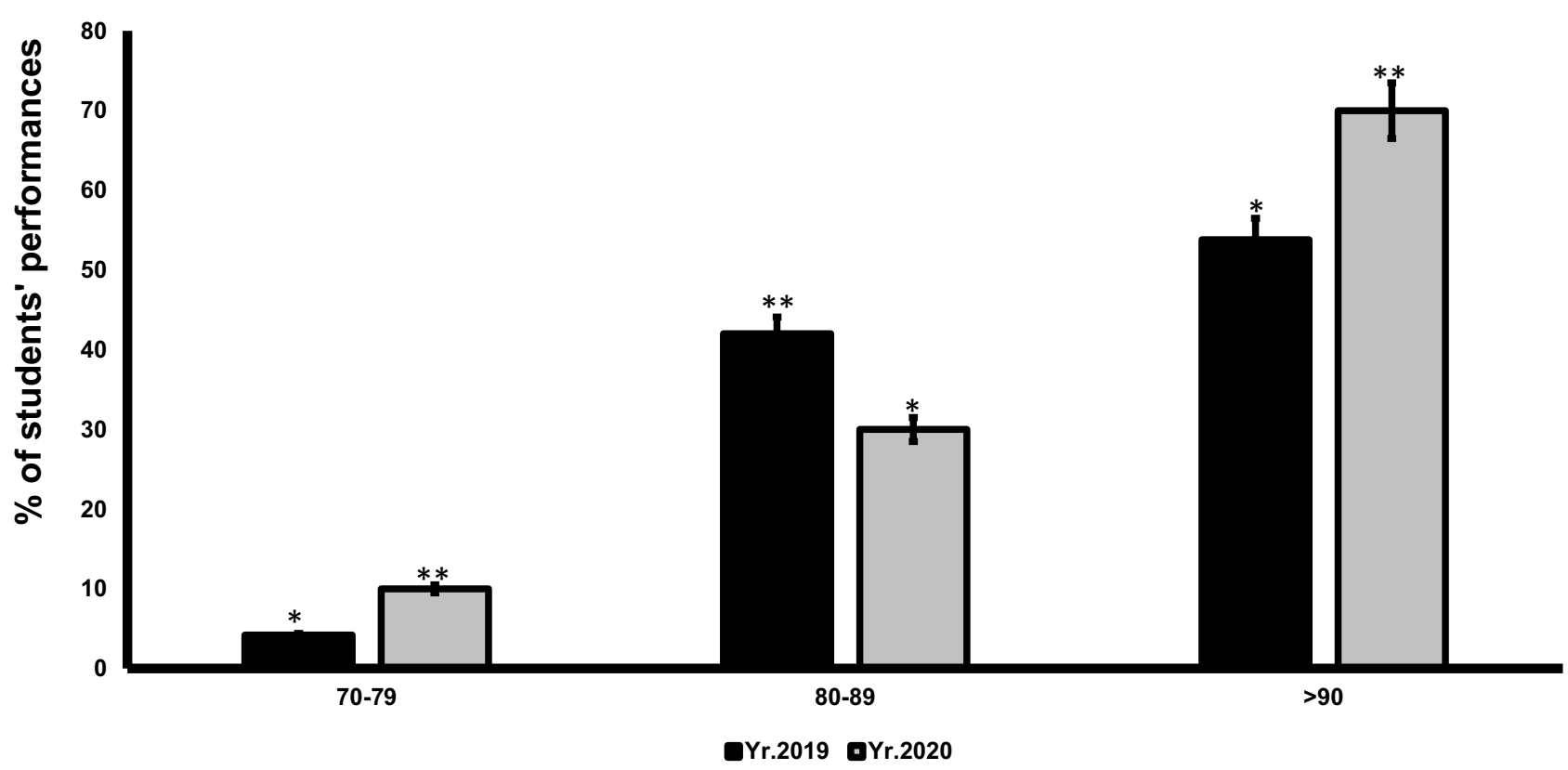

Fig. 4 Student performance in grade categories for $70-79 \%, 80-89 \%$, and $>90 \%$. Differences between students of different grade levels were determined with ANOVA with repeated measures for all variables with homogenous variance across groups. Post hoc analysis for all significant differences found by ANOVA between different student

provide virtual support for their learning. In general, the adaptive learning model generated personalized learning materials that helped medical students to improve mastery of learning neuroscience laboratory activities and virtually connected students to faculty members. This type of model helped faculty members to interact with medical students at a much deeper level to address student concerns and to facilitate their learning process [38-40].

A major finding in this study is that in the adaptive blended learning, more students scored between 80-89\% and $70-79 \%$, and fewer students scored $>90 \%$ when compared with the in-person learning of neuroscience laboratory activities. A major factor that appeared to encourage the effectiveness of the adaptive blended learning for medical students is that it blends digital technology with online activities. Medical students that are trained in an integrated medical curriculum are very effective in using digital technology [13-15]. They are also familiar with self-study and working independently at their own pace $[16,17]$. The effortlessness with which medical students explore digital technologybased learning [41, 42], along with the independence they have developed within the integrated medical curriculum, made the transition to an adaptive blended learning a little less stressful [11, 43-47].

While the adaptive blended learning provides an autonomy of independent learning, self-study approach that allows grade levels. A Tukey post hoc analysis followed each significant result from a Fisher's ANOVA, while a Games-Howell post hoc analysis followed each significant result from all post hoc analysis from a Welch's ANOVA as noted in Fig. 4. *, $* * P<0.05$

for a fluid schedule for students to work at their own pace, it broadened our medical student performance such that more students scored between 80 and ' $90 \%$. By contrast, in-person learning skewed the student performance and identified high performing students (scoring $>90 \%$ ) in medical neuroscience laboratory activities. An important finding in this study is that more students scored between 70 and $79 \%$ in the adaptive blended learning approach compared with fewer students in the in-person approach. Therefore, while the adaptive blended learning provided greater flexibility for independent learning, self-study approach, it is possible that this non-traditional learning format was probably not as suitable for the $<10 \%$ medical students that scored between 70 and $79 \%$. These students might well have performed better using the in-person interactive laboratory activities to process learned materials.

While adaptive blended learning provides an individualized learning experience with technologies focused on the learners' strengths $[48,49]$, we recognized that medical students have different levels of depth in understanding digital technology [50]. While some may achieve competency in using digital technology relatively quickly, others may struggle to do so [51, 52]. Variation in competency in using digital technology is well known among medical students, but the problem may not easily be solved when learning materials are taught in the traditional classroom. 
Adaptation in our adaptive learning approach is not just limited to ensuring that materials are delivered according to the needs of the medical students and during the COVID-19 pandemic. Other forms of adaptation were considered including their strengths and capability in the use of adaptive digital technology that medical students could easily navigate the contents of the neuroscience laboratory materials and implement virtual interactive activities-such as using the WebEx/Panopto/Canvas for the discussions to connect peers and faculty with each other. Therefore, in our adaptive blended learning model, we provided an adaptive environment of an individualized learning experience using digital technology as a strength of medical students to enhance faculty-student interactions, and student-content interactions. This model recognized medical students' strengths and ensured that learning materials were learner specific, and the adaptive learning platform helped individual medical students to better utilize their competency strengths in learning the content materials of the neuroscience laboratory activities during this pandemic.

\section{Limitations}

While our data indicated that students performed better in the wet lab in person when compared with online, future studies are needed on whether personal interactions with clinicians during the wet lab and clinical cases discussion might facilitate such a better performance. Our results indicate that an adaptive flexible blended learning approach that combined virtual face-to-face instruction using digital technology with online learning of neuroscience laboratory activities provided a unique educational experience for Year 1 medical students to learn neuroscience laboratory activities during the COVID-19 pandemic. It is also important to implement the model in other courses such as anatomy and histology to compare the performance of students in neuroscience and other courses. Therefore, a future study on the adaptive flexible blended learning approach model in anatomy or histology courses is worth pursuing. Although students reported positive impact of the model in their evaluation, collecting qualitative and quantitative feedback from students is another limitation. Also, feedback was not collected from academic staff who participated in the sessions. Results from academic staff members with positive feelings about adaptive flexible blended learning approach would have provided the perspective of the instructors concerning the positive impact produced by the use of the model. The use of proctoring software and remote surveillance may have had an effect on students' performance. A future study to determine whether the remote surveillance may affect students' performance will inform medical educators about the effects of remote proctoring on students' performance in online learning.

\section{Conclusion}

In the adaptive blended learning approach, we used a digital technology platform to create a virtual interaction that replaced face-to-face interaction with students for clinical case discussions sessions and to facilitate interactive discussions for swift tutor feedback and guided learning. We combined this modality with learning of neuroscience laboratory activities to provide a unique educational experience for year one medical students during the time of a global pandemic. Evaluation of student performance indicated that the method offered medical students the appropriate opportunity to learn neuroscience laboratory activities required in their medical professional education in a challenging time with a pandemic disease, where the opportunity for in-person learning is difficult to implement. Compared with traditional learning, online learning has its advantages to enhance students' learning and should be an important component in designing an integrated medical curriculum. To enhance its effectiveness, digital learning materials, learning goals, and students' preferences should be considered.

\section{Declarations}

Ethical Approval This study was approved by the institutional review board of the Health institutional committee for ethics.

Conflict of Interest The authors declare that they have no conflict of interest.

\section{References}

1. Gregory SR, Cole TR. The changing role of dissection in medical education. Jama-Journal of the American Medical Association. 2002;287:1180-1.

2. Ghosh SK. Cadaveric dissection as an educational tool for anatomical sciences in the 21 st Century. Anat Sci Educ. 2017;10:286-99.

3. Clark RM, Kaw A. Adaptive learning in a numerical methods course for engineers: evaluation in blended and flipped classrooms. Comput Appl Eng Educ. 2020;28:62-79.

4. Fazal M, Panzano B, Luk K. Evaluating the Impact of blended learning: a mixed-methods study with difference-in-difference analysis. TechTrends. 2020;64:70-8.

5. Phillips A, Pane JF, Reumann-Moore R, Shenbanjo O. Implementing an adaptive intelligent tutoring system as an instructional supplement. Etr\&D-Educational Technology Research and Development. 2020;68:1409-37.

6. Saric-Grgic I, Grubisic A, Seric L, Robinson TJ. Student clustering Based on learning behavior data in the intelligent tutoring 
system. International Journal of Distance Education Technologies. 2020;18:73-89.

7. Sharma N, Iain D, Dong C. Adaptive learning in medical education: The final piece of technology enhanced learning? Ulster Med J. 2017;86:198-200.

8. Bransford JD, Brown AL, Academy N, Press W, D.C. . How people learn: brain, mind, experience, and school; 1999.

9. German A, Gallego T. Adaptive, active and collaborative learning through blended learning. In: Chova LG, Martinez AL, Torres IC, eds. Edulearn 16: 8th International Conference on Education and New Learning Technologies, 2016; 1750-1760.

10. Hernandez Y, Ramirez MP. Adaptive and blended learning for electrical operators training with virtual reality systems; 2016.

11. Tashiro J, Hebeler A. An adaptive blended learning health education model for families of a parent with serious medical problems. In: Cheung SKS, Lee LK, Simonova I, Kozel T, Kwok LF, eds. Blended learning: Educational innovation for personalized learning, Icbl 2019, 2019; 59-71.

12. Wangwattana B, Lertnattee V. Adaptive content development for blended Learning in microscopic of herbal medicines. In: Cheung SKS, Lee LK, Simonova I, Kozel T, Kwok LF, eds. Blended learning: Educational innovation for personalized learning, Icbl 2019, 2019; 137-148.

13. Jameson DG, Hobsley M, Ohanlon P, Buckton S. Real-time interactivity on the SuperJANET network. Interact Comput. 1996;8:285-96.

14. Marchevsky AM, Relan A, Baillie S. Self-instructional, "virtual pathology" laboratories using web-based technology enhance medical school teaching of pathology. Hum Pathol. 2003;34:423-9.

15. McCutcheon K, Lohan M, Traynor M, Martin D. A systematic review evaluating the impact of online or blended learning vs. face-to-face learning of clinical skills in undergraduate nurse education. Journal of Advanced Nursing. 2015;71:255-270.

16. Brechtel L, Womack L, Ubah C, et al. Early Clinical experience in a year one medical neuroscience course enhances students' performance. Medical Science Educator. 2018;2:315-26.

17. Brechtel L, Wormack L, Frazier A, et al. Middle school students: active learning activities for stroke education and healthy lifestyles. J Child Fam Stud. 2019;28:1955-64.

18. Isayeva O, Shumylo M, Khmilyar I, Myskiv I, Mylyk O. Blended learning in higher medical education: principles and strategies of teaching foreign languages. Advanced Education. 2020:11-18.

19. Micheal S, Marjadi B. Blended learning to teach gender in medical school. Clinical Teacher. 2018;15:208-13.

20. Swito YS, Koesoema AP, Pribadi A. Development of a blended and eLearningc course on anatomy and physiology for engineers in Indonesia: Lessons learned and future developments. In: Lhotska L, Sukupova L, Lackovic I, Ibbott GS, eds. World congress on medical physics and biomedical engineering 2018, Vol 1, 2019; 881-884.

21. Brown MG. Blended instructional practice: a review of the empirical literature on instructors' adoption and use of online tools in face-to-face teaching. Internet and Higher Education. 2016;31:1-10.

22. Bates AW. Teaching in the digital age: Guidelines for designing teaching and learning. Vancouver Tony Bates Associates; 2015.

23. Graham CR. Emerging practice and research in blended learning. In M. G. Moore (Ed.), Handbook of distance education (3rd ed., pp. 333-350). New York, NY: Routledge. New York Routledge; 2013.

24. Protsiv M, Atkins S, Consortium A. The experiences of lecturers in African, Asian and European universities in preparing and delivering blended health research methods courses: a qualitative study. Global Health Action. 2016;9.
25. Ramklass SS. The transition from traditional lectures to blended learning: piloting of an e-learning research methods module for postgraduate medical studentS. In: Chova LG, Martinez AL, Torres IC, eds. Edulearn14: 6th International Conference on Education and New Learning Technologies, 2014; 400-404.

26. Saupe JL. Some useful estimates of the kuder-richardson formula number-20 reliability coefficient. Educ Psychol Measur. 1961;21:63-71.

27. Zimmerman DW, Burkheimer GJ. Coefficient alpha, test reliability, and heterogeneity of score distributions. Journal of Experimental Education. 1968;37:90-5.

28. Mattick K, Knight L. The importance of vocational and social aspects of approaches to learning for medical students. Adv Health Sci Educ. 2009;14:629-44.

29. Meguid EMA, Khalil MK. Measuring medical students' motivation to learning anatomy by cadaveric dissection. Anat Sci Educ. 2017;10:363-71.

30. Mak-van der Vossen M, Teherani A, van Mook W, Croiset G, Kusurkar RA. Investigating US medical students' motivation to respond to lapses in professionalism. Medical Education. 2018;52:838-850.

31. Barte JCM, Nieuwenhuys A, Geurts SAE, Kompier MAJ. Motivation counteracts fatigue-induced performance decrements in soccer passing performance. J Sports Sci. 2019;37:1189-96.

32. Groening C, Binnewies C. "Achievement unlocked!"- the impact of digital achievements as a gamification element on motivation and performance. Comput Hum Behav. 2019;97:151-66.

33. Hangen EJ, Elliot AJ, Jamieson JP. Highlighting the difference between approach and avoidance motivation enhances the predictive validity of performance-avoidance goal reports. Motivation and Emotion. 2019;43:387-99.

34. Jimenez EJB, Villegas JYV, Panduro JVR. The methodologies, intrinsic motivation and performance of the students of the Faculty of Education of the UNMSM. Dilemas ContemporaneosEducacion Politica Y Valores. 2019;6.

35. Law KMY, Geng S, Li TM. Student enrollment, motivation and learning performance in a blended learning environment: the mediating effects of social, teaching, and cognitive presence. Comput Educ. 2019;136:1-12.

36. Wenzel AK, Krause TA, Vogel D. Making performance pay work: the impact of transparency, participation, and fairness on controlling perception and intrinsic motivation. Review of public personnel administration. 2019;39:232-55.

37. Zou JH, Liu K, Han LL. The impact of a rich media platform to table tennis learners' performance and participation motivation. Interactive Learning Environments.

38. Branzetti J, Gisondi MA, Hopson LR, Regan L. Aiming beyond competent: the application of the taxonomy of significant learning to medical education. Teach Learn Med. 2019;31:466-78.

39. Carney PA, Mejicano GC, Bumsted T, Quirk M. Assessing learning in the adaptive curriculum. Med Teach. 2018;40:813-9.

40. Hudder A, Tackett S, Moscatello K. First-year experience Implementing an adaptive learning platform for first- and second-year medical students at the Lake Erie College of Osteopathic Medicine. J Am Osteopath Assoc. 2019;119:51-8.

41. Wang YP, Han XB, Yang J. Revisiting the blended learning literature: using a complex adaptive systems framework. Educational technology \& society. 2015;18:380-93.

42. Wickremaratne J, Wimalaratne $\mathrm{G}$, Goonetilleke V, Ieee. A blend of adaptive and digital learning towards language proficiency; 2008.

43. Ferreira P, Gomes TT, Severo M, Ferreira MA. Assessment of the effect of digital education interventions in medical education. In: Chova LG, Martinez AL, Torres IC, eds. Iceri2014: 7th 
International Conference of Education, Research and Innovation, 2014; 3655-3663.

44. Harden RM. The virtual learning enviroment in medical education-past, present and future; 2010.

45. Muhsin HK, Ieee. The using of E-learning techniques to improve the medical education; 2008.

46. Taveira-Gomes T, Saffarzadeh A, Severo M, Guimaraes MJ, Ferreira MA. A novel collaborative e-learning platform for medical students-ALERT STUDENT. Bmc Medical Education. 2014;14.

47. Trukhacheva N, Pupyrev N. Blended Learning strategy in the altay state medical university. In: Blobel B, Engelbrecht R, Shifrin MA, eds. Large Scale Projects in Ehealth: Partnership in Modernization, 2012; 72-75.

48. Greving S, Lenhard W, Richter T. Adaptive retrieval practice with multiple-choice questions in the university classroom. Journal of Computer Assisted Learning.

49. Wade SWT, Moscova M, Tedla N, et al. Adaptive tutorials versus web-based resources in radiology: a mixed methods analysis of efficacy and engagement in senior medical students. Acad Radiol. 2019;26:1421-31.
50. Hyll M, Schvarcz R, Manninen K. Exploring how medical students learn with the help of a digital presentation: a qualitative study. Bmc Medical Education. 2019;19.

51. Lakhal S, Mukamurera J, Bedard ME, Heilporn G, Chauret M. Features fostering academic and social integration in blended synchronous courses in graduate programs. International Journal of Educational Technology in Higher Education. 2020;17.

52. Regmi K, Jones L. A systematic review of the factors-enablers and barriers-affecting e-learning in health sciences education. Bmc Medical Education. 2020;20.

53. Leichnetz GR. Digital neuroanatomy:. In: Leichnetz GR (Editor). Richmond, 2006.

Publisher's Note Springer Nature remains neutral with regard to jurisdictional claims in published maps and institutional affiliations. 